\title{
The Effects of Physical and Mac Parameters on the Routing by Cross- Layers Interaction Approach
}

\author{
Ouchker Elmekki, University Hassan II, Casablanca, Morocco \\ Abderrahim Maizate, University Hassan II, Casablanca, Morocco \\ Mohammed Ouzzif, University Hassan II, Casablanca, Morocco
}

\begin{abstract}
Wireless sensor networks (WSN) are an ad hoc network consisting of miniaturized autonomous sensors that communicate with each other over a radio link. In this paper, the authors' contribution is a cross-layer approach. To achieve this approach, the authors proposed in the first part an improvement of LEACH algorithm named WEIGHTED_LEACH. This algorithm is an enhancement of LEACH by inserting novel factors in the threshold equation in order to choose the best node to be $\mathrm{CH}$ by competitiveness strategy. In the second part, the authors propose a new adaptable cross-layer design realized by proposed algorithm and AODV protocol with cross layer interaction algorithm. This is an improvement by the control of data fusion in the network using multi-hop routing based on weight metric deducts from relative parameters of node such RSSI, SINR, residual energy, and distance. A comparative analysis between WEIGHTED_LEACH and LEACH, shown for different configurations the efficiency of the proposition in terms of energy saving and life time of WSN cluster, are evaluate under the NS2 simulator.
\end{abstract}

\section{KEYWORDS}

AODV, Distance, Energy, Leach, Ns2.35, RSSI, SINR, WSN

\section{INTRODUCTION}

In recent years, with the rapid development of communication and sensor technologies, Wireless Sensor Networks (WSN) are emerging in many industrial and consumer area.

(WSN) is a group of static sensor nodes which gather information and deliver to the base station. In WSN sensor nodes deployed to monitor various physical and environmental parameters. Due to fast development in mobile Internet technology, mobile wireless sensor network (MWSN) has become a popular field of research and replacement of static WSN. 
MWSN is a collection of mobile sensor nodes that aim to sense data from the environment and effectively deliver the base station.

MWSN can be classified into three types, which are as follows:

- Mobile base station and mobile sensor;

- Mobile base station and static sensor;

- Static base station and mobile sensor.

\section{BACKGROUND}

\section{Wireless Sensor Environments}

Wireless sensor network (WSN) is a developing technology that improves the resource utilization in various fields such as home automation, e-health, smart grid, precision agriculture etc. It consists of sensor devices, which works autonomously with its sensing, communication and computation capabilities. In most of the sensor network applications the nodes will be deployed in remote areas, so replacing the battery often is impossible in many WSN scenarios. Hence energy is considered as a valuable resource in resource constrained wireless sensor networks.

WSN communication suffers from various problems with Self-organization nodes, and environment obstacles. To ensure the best performance of network it is necessary to try to solve the problems of unreliability of links, and to avoid successive retransmission of packet in order to reduce the energy consumption at each node to its task of routing in other words minimizing the route failed re-selection.

Consequently, the evaluation specifies the quality of link, minimize the errors and make the interchangeability between nodes more effective to solve the problems imposed by the unreliability of links. To overcome these problems of layers modules, most researchers proposed cross layer design.

The aim of Cross-layer design is to improve the performance of all layers and share key parameters between these layers.

Whole system performance can be improved if the MAC can get parameter from the PHY about when and how the noise level is changing, as a result the MAC can schedule transmission during the periods of time in which noise levels are lower.

All protocols proposed in WSN have to take in their account the basic inherent characteristics of the network which are: dynamic topology, variable link capacity and bandwidth constraints, energy constraints nodes and multi-hop communications. All these characteristics are seriously challenged the OSI layer design which is characterized by the modularity (Kawadia \& Kumar,2005), and permit to create a new methodology named Cross-layer. Cross-layer design refers to an optimized approach design done by allowing layers to exchange state parameter in order to obtain performance gains. The sharing of parameter enables each layer to have global pictures of the constraints and characteristics of the network. Basically, the cross-layer design enables the network protocols and the applications to observe and respond to the changing networks and channel conditions (Rappaport \& al,2002).

We can classify the shared parameter of layers as:

- Physical Layer Parameters (PLPs): By focusing on the effects of link quality, power control, a harmonization between Mac and physical layer is investigated. For example, given the current channel state condition, the MAC protocol may adjust some parameters in order to reduce the energy consumption.

- Mac Layer Parameters (MLPs): The link layer has notification mechanisms in case of absence of the acknowledge packet or the clear to send (CTS) packet which permit the network protocols to research newer routes and update their routing table. 
- Network Layer Parameters (NLPs): In the route discovery process the network layer can use the parameter of the receive signal strength to choose reliable links to form stable routes by monitoring the signal quality to judge whether the neighbors are approaching or leaving $(B$ Ramachandran \& S Shanmugavel, 2008).

- The session layer parameters (SLPs), the transport layer parameters (TLPs) and the application layer parameters (ALPs) of the OSI model are not considered in our work.

\section{Cross Layer Adaptation Approach}

By exploiting cross-layer interactions, researchers hope to develop a new framework that will help to solve some of the difficulties inherent in WSNs.

Recent research into cross layer design has looked to optimize different aspects of the WSN; these approaches have been:

- Using cross layer design to improve the power efficiency of WSNs, thereby increasing the network lifetime;

- Optimizing system throughput in hopes of making the problem of scalability of WSNs tractable;

- Satisfying QoS requirements of WSNs;

- Improving resource efficiency.

\section{Quality of Service in Cross-Layer Approach}

QoS routing under severe constraints is a mechanism that creates paths in the WSN, then selects and maintains paths satisfying QoS requirements for the packet flows, subject to residual power reserves at nodes and along paths, based on the available network "quality."

It groups resource parameters and performance metrics associated with protocol layers, i.e., the application, network, link/medium access control (MAC), and physical (PHY) layers, then maps them to data classes.

(Braden, Clark \& Shenker, 1994), (Blake, Black, Carlson, Davies, Wang \& Weiss, 1998), (Nikaein, Bonnet, Moret, Rai, 2002), \& (Nikaein, Bonnet, 2003) these approach is suggested by the strict dependence of QoS on network "quality," i.e., interaction and stability of resources on routes and at intermediate nodes. Some QoS metrics are expressed in terms of interrelated parameters, e.g., RSSI, SINR...

According to (Acampo \& Naghshineh, 1994), QoS metrics to which they have the highest correspondence, is indicated in Figure 1.

Quality in the physical layer is correlated to transmit power, channel code selection, packet rate, modulation type, and antenna mode. As physical layer parameters (PLPs), these influence battery conservation and network lifetime, either directly or indirectly.

In the Network layer, Quality is related to Network-layer functions include fast sensor-to-sink path reservation, packet store and forwarding, congestion control such as low-priority packet dropping, and other routing and flow control tasks. According to (Hortos,2009), mapping from QoS metrics and energy-related metrics to the different layers is very important in cross layer approach.

\section{MAIN ISSUE AND SOLUTIONS}

\section{Topology Issue}

In MWSN, the mobility of nodes, the obstacles and the environment conditions produces frequent changes of topology and unmanaged traffic flow which increases consequently link break in the active path and dropping of packets due to queue overflow. 
Figure 1. QoS metrics

\begin{tabular}{|c|c|}
\hline Protocol Stack & Quos Metrics \\
\hline Application Layer & $\begin{array}{c}\text { Application Requirements } \\
\text { Packet Classes } \\
\text { Application } \uparrow \text { Layer Parameters } \\
\text { Throughput }\end{array}$ \\
\hline Network Layer & $\begin{array}{c}\text { Network Layer Parameters } \\
\text { Quality PathlConnectivity } \\
\text { Queue Capacity, Power, Link reliability, Hop count }\end{array}$ \\
\hline Mac Layer & $\begin{array}{c}\text { Mac } \\
\text { SINR,ETX,RSSI, Bandwidth }\end{array}$ \\
\hline Physical Layer & $\begin{array}{c}\text { Physical } \downarrow \text { Layer Parameters } \\
\text { Modulation, Transmission Power, Packet Rate, Duty Cycle }\end{array}$ \\
\hline
\end{tabular}

The motivation for this research work arises from the knowledge gained by literature review and scope of improving WSN clusters by suggesting some changes to the clustering process with introducing competitiveness approach in the first, suggesting some changes to the path discovery process of AODV for improving reliability and efficiency of mobile WSN in the second, and proposing cross layer algorithm to collaborate layers protocols in the end.

\section{Solutions and Recommendations}

\section{Protocol Design and Implementation}

LEACH (low-energy adaptive clustering hierarchy) is an energy efficient clustering-based protocol (Limin, Jianzhong \& Chen,2005), Clustering process is executed continuously and circularly in LEACH, and each round can be divided into two stages: The set-up stage of clusters; the steady-state stage for data transmission clusters. In the set-up stage, it would generate one or more of random cluster heads based on the threshold T(n) in formula (1), and each cluster is formed dynamically by the adjacent nodes. In steady-state phase, cluster nodes send data to cluster heads. Then the data will be sent to the base station after data fusion. The whole process is completed. LEACH protocol can ensure the probability of each node as a cluster head, so the energy consumption of the network is relatively balanced:

$$
T(n)=\left\{\begin{array}{lr}
\frac{P}{1-P x\left[r \bmod \left(\frac{1}{P}\right)\right]} & n \in G \\
0 & n \notin G
\end{array}\right.
$$

In formula (1), $P$ represents percentage of the cluster head in all nodes; $r$ indicates rounds of the elections; $\operatorname{rmod}(1 / \mathrm{P})$ explains the number of nodes of the cluster head is elected in a cycle; $\mathrm{G}$ represents node collection which has not been elected as cluster head in this round.

(SHAN, DONG, LIAO, SHAO, GAO and Yang, 2013) present an improvement on LEACH protocol by adding factors of distance and energy on threshold equation to increase the life cycle of network. 


\section{LEACH protocol has certain disadvantages:}

- In the election of cluster heads and the establishment of clusters, the residual energy of clusterhead nodes is not considered. If you select the less energy nodes as the cluster head, it greatly reduces the network life cycle;

- Cluster heads communicate with the base station directly. The farther away the distance between heads is, the more energy the nodes will consume.

The protocol is proposed to overcome limitations of conventional LEACH to improve the life time and reduce the energy consumption of the WSN. The proposed enhancement is achieved by inserting novel factors in the threshold equation in order to choose the optimum node having high resources to be Cluster Head $(\mathrm{CH})$. The novel proposed parameters to be inserted are residual energy of node, distance between node and base station, the Received Signal Strength (RSSI) which is related to the communication pass distance and SINR that reflect the effect of communication channel noise and interference.

\section{The Improved Leach: weighted_leach}

\section{Protocol Design Metrics}

The SINR, RSSI, distance and residual energy are used for Improvement of competitiveness in $\mathrm{CH}$ election.

\section{SINR Metric}

SINR (signal to-noise ratio) is an important parameter in the physical layer of the network which measures the quality of a link in WSN. Network nodes that have the best SINR with the base station should have the priority to be $\mathrm{CH}$, as this will decrease the probability of link failure during sending or receiving data.

SINR on the WSN operation is embedded as a ratio with threshold formula of LEACH classic indicated in formula (1).

\section{RSSI Metric}

In practical WSN, obstacles and environments condition can be decrease data transmission using radio transmission power RSSI of a node is one of the parameters that should be added to threshold formula (1) of LEACH classic.

Most researchers take the distance between two nodes as the effective factor in energy consumption but this is not true in all situations, because if there is bad environment condition or obstacles inside WSN, RSSI will be low. For this reason, RSSI is more take in account. The node with the best RSSI to Base station are high chance to save more energy in the transmission of its collected data to base station and to avoid receiving and sending failure.

\section{Residual Energy}

(Bayarkli, Erdogan, 2012) have used this parameter during the CHs selection phase to balance the load (energy consumption) between nodes in order to provide a stable structure and to increase the network lifetime. According to paper (Sheta, Mahmoud, Abdelwahab, Elaraby, 2015), The main constraint that affects the practical design of Wireless Sensor Networks (WSNs) is the network energy as network nodes has limited resources capabilities. (Sheta, Mahmoud, Abdelwahab, Elaraby, 2015) proposed enhancement on the conventional LEACH by inserting a remaining or consumed energy as factor to the conventional threshold equation. This factor of each node make the selection of $\mathrm{CH}$ depends on the nodes energy status information. $\mathrm{CH}$ candidate that have the high energy assure an enhancing in network life time. 


\section{Distance}

The distance between nodes is also a very important criterion in the CHs selection phase. This parameter influences the transmission power and the number of hops to reach the destination and, consequently, the energy used to make the transmission.

\section{Optimized Threshold Equation for $\mathrm{CH}$ Selection}

According to formula of paper (Jian \& Dandan, 2013), we have considered other parameters like the residual energy of nodes, the RSSI, the SINR and the distance between the node and the base station when we select the cluster head, our improved threshold T'(n) expression:

$$
T^{\prime}(n)=\left\{\begin{array}{lc}
T(n) *_{\text {weight_metric }} & n \in G \\
0 & \text { otherwise }
\end{array}\right.
$$

where:

$$
\text { weight_metric }=\left(w 1 * \frac{E_{n-c u r}}{E_{t-c u r}}+w 2 * R S S I+w 3\right)
$$

$\mathrm{E}_{\mathrm{n}-\mathrm{cur}}$ represents residual energy node in the current; $\mathrm{E}_{\mathrm{t}-\mathrm{cur}}$ stands for all nodes in the rest of the total energy in a network; $\mathrm{d}_{\max }$ indicates the maximum distance between the node and the base station; $\mathrm{d}_{\mathrm{ns}}$ explains the distance between node and the base station; $\mathrm{d}_{\min }$ represents the minimum distance between node and the base station; RSSI represents the strength of the received RF signal of a node, SINR represents the signal to-noise ratio.

$\mathrm{w}_{1}, \mathrm{w}_{2}, \mathrm{w}_{3}$ and $\mathrm{w}_{4}$ are constants weight indicating the importance of its relevant factor. The summation of these constant weights is unity.

Main Algorithm

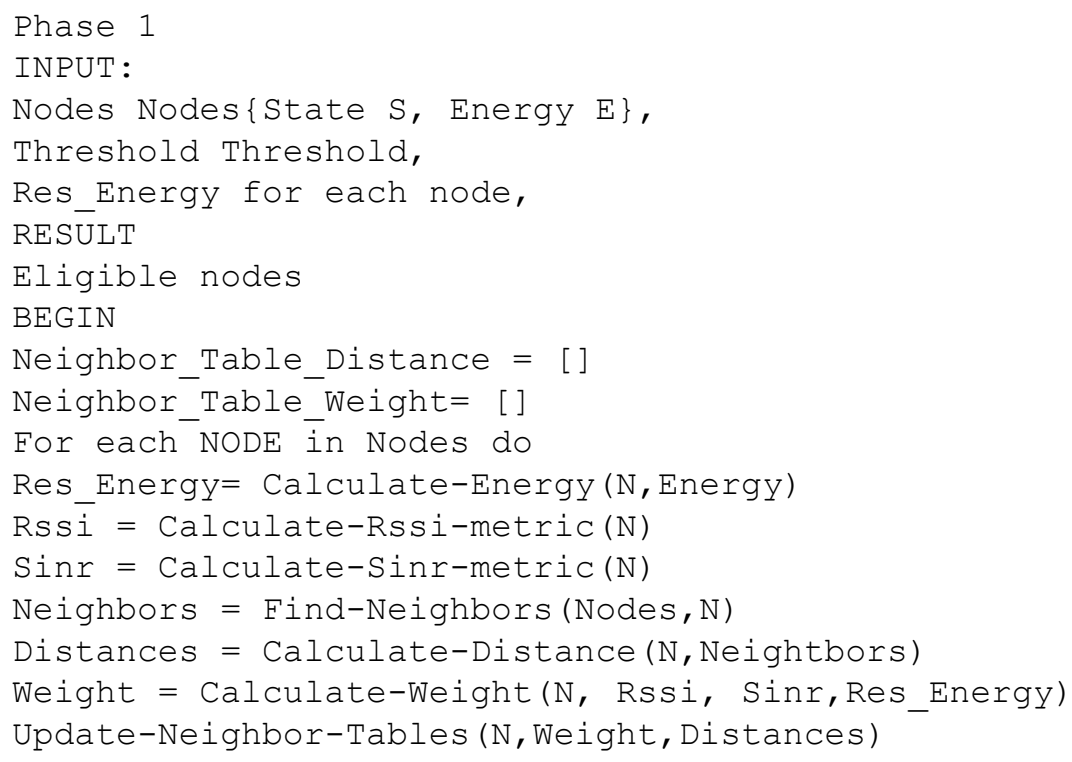




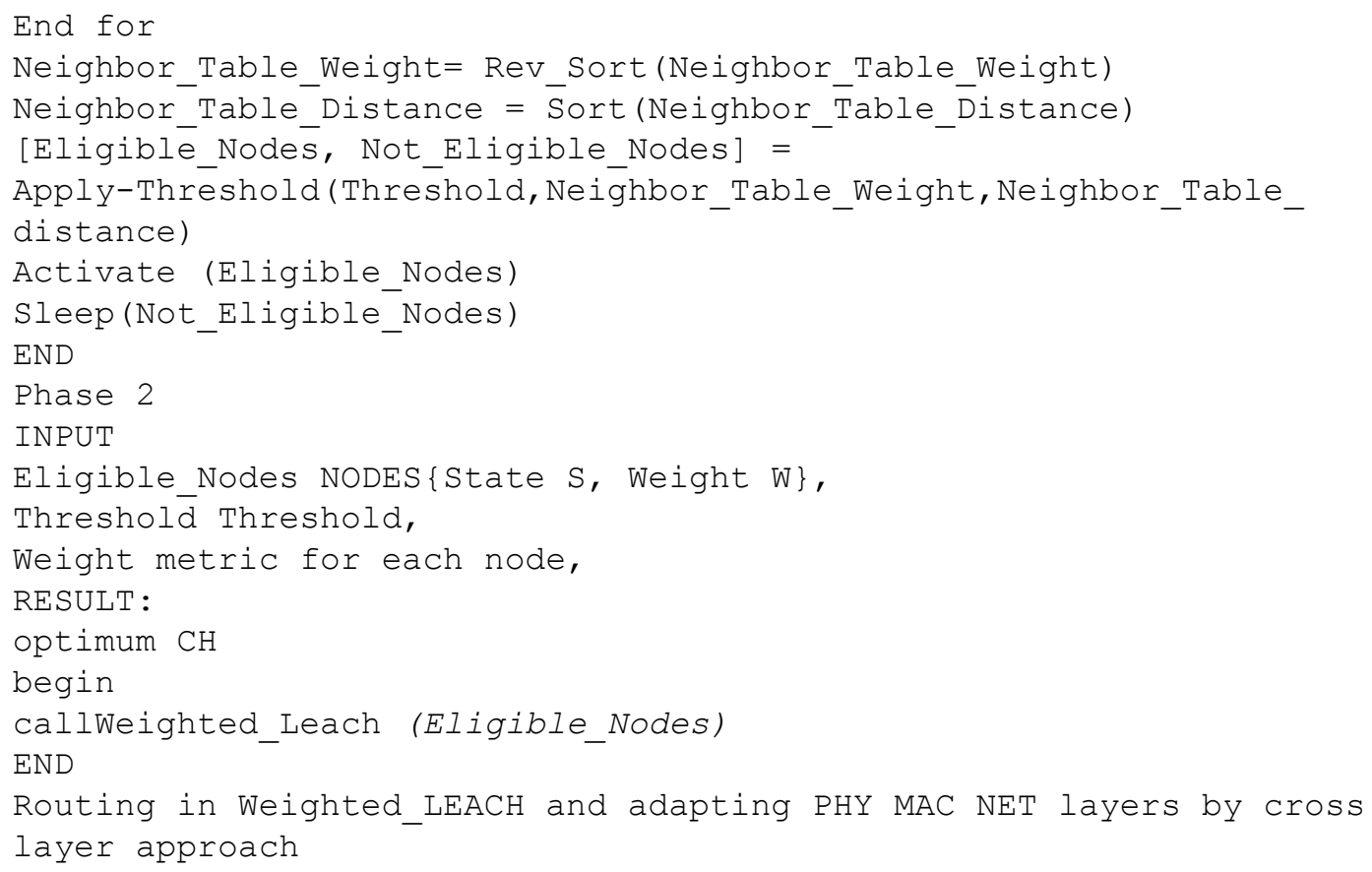

The cross-layer design is indicated in Figure 2.

In our proposed cross layer, we have modified the procedures at physical layer to pass the computed values of RSSI, SINR, and residual energy to the MAC and network layers. But before passing these parameters to the upper layers, it is necessary to gather them in one metric calculated by the formula 2 as follows:

$\mathrm{f}($ weight_metric $)=\operatorname{Max}($ weight_metric $)$

The computed weight metric are passed to routing layer along the routing control packets, where it is stored in routing/neighbor tables and used in some decision making process.

Figure 2. Cross Layer design

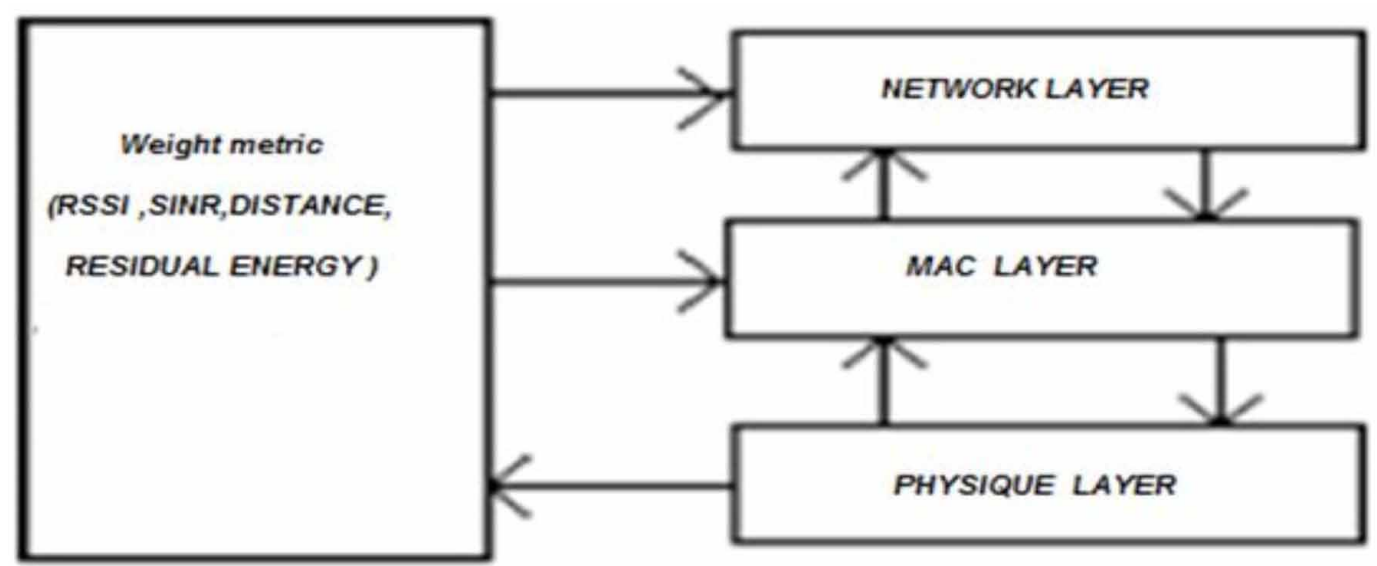




\section{Cross Layer Interaction Approach}

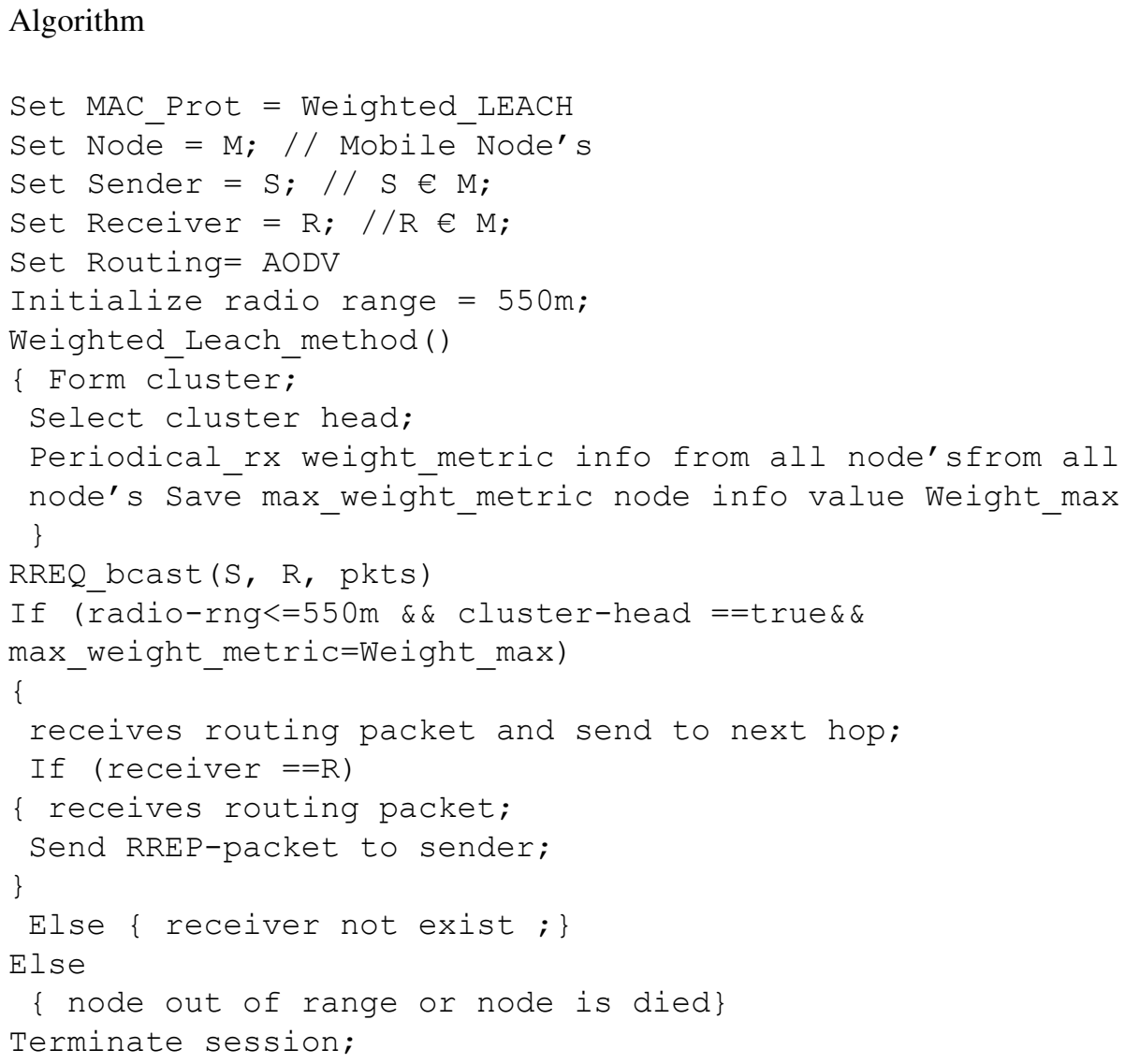

\section{SIMULATION AND RESULTS}

Simulation of the proposed weighted_Leach was done using ns 2.35 simulator simulation area $(50 \times 50)$ $\mathrm{m}^{2}$ with BS located at the middle of that area. The detail parameter setting for the simulations is indicated in Table 1 and 2.

\section{Scenario 1: Impact of RSSI}

In this scenario, for one period of simulation, we fix the SINR metric, we vary the RSSI metric and we measure energy.

Simulation results for Number of alive node and Network residual energy with various round numbers is indicated in Figure 3.

\section{Scenario 2: Impact of SINR}

In this scenario, for one period of simulation, we fix the RSSI metric, we vary the SINR metric and we measure energy.

Simulation results for Number of alive node and Network residual energy with various round number is indicated in Figure 4. 
Table 1. Simulation parameters

\begin{tabular}{|l|l|}
\hline \multicolumn{1}{|c|}{ Parameters Description } & \multicolumn{1}{c|}{ Values } \\
\hline Number of Round & 1000 \\
\hline Number of nodes & 100 \\
\hline Initial energy of node & $0.5 \mathrm{~J}$ \\
\hline En_cur & $50 \mathrm{~nJ}=$ bit \\
\hline Energy Aware & Leach,weighted_leach \\
\hline Routing Protocol & AODV \\
\hline Traffic model & CBR \\
\hline Packet size & 512 bytes \\
\hline Mobility model & Random way point \\
\hline
\end{tabular}

Table 2. Different Set of $W_{i}$ values used in formula (1)

\begin{tabular}{|l|l|l|l|l|}
\hline \multicolumn{1}{|c|}{ Set } & \multicolumn{1}{c|}{ Energy Factor } & \multicolumn{1}{c|}{ RSSI } & \multicolumn{1}{c|}{ SINR } & \multicolumn{1}{c|}{ Distance } \\
\hline Set 1 & 0.30 & 0.30 & 0.30 & 0.10 \\
\hline Set 2 & 0.3 & 0.2 & 0.4 & 0.1 \\
\hline Set 3 & 0.4 & 0.3 & 0.1 & 0.2 \\
\hline Set 3 & 0.1 & 0.4 & 0.3 & 0.2 \\
\hline
\end{tabular}

Figure 3. Simulation results for number of alive nodes and network residual energy with various round numbers
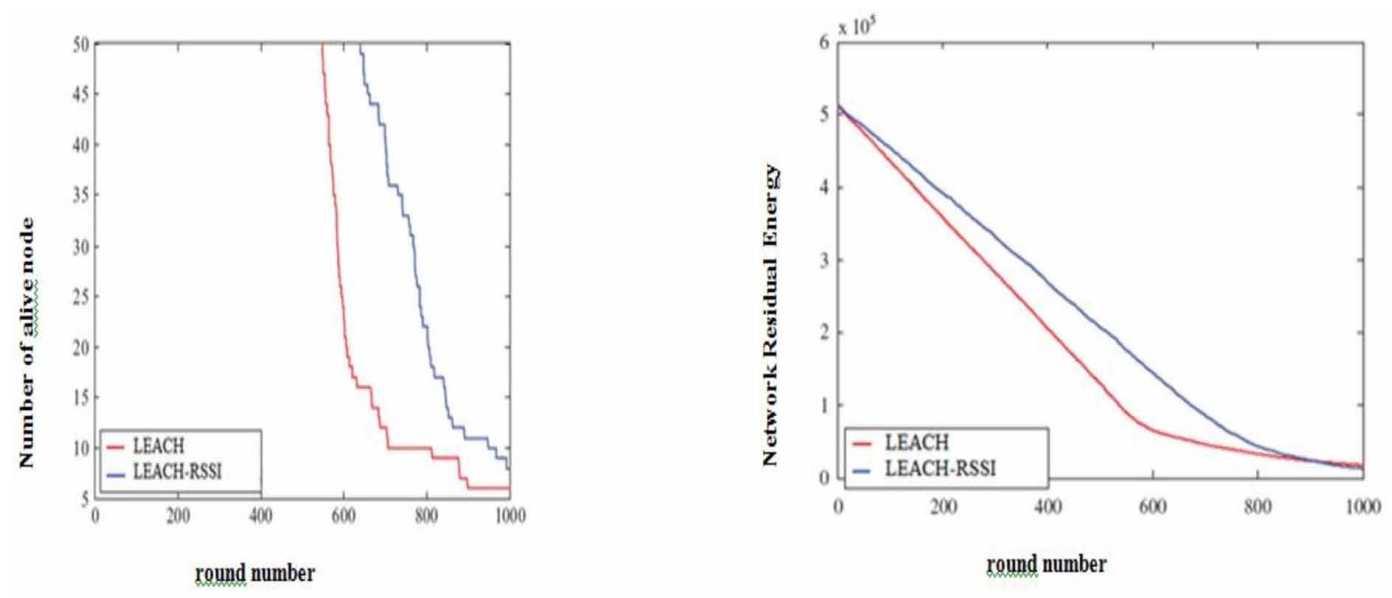

The embedded factor RSSI and SINR as shown in the figures has a great effect on the network operation as it increases the number of alive nodes and residual energy of nodes. Therefore, the addition of RSSI and SINR to threshold equation improves the network lifetime by compared to LEACH. 
Figure 4. Simulation results for number of alive notes and network residual energy with various round numbers
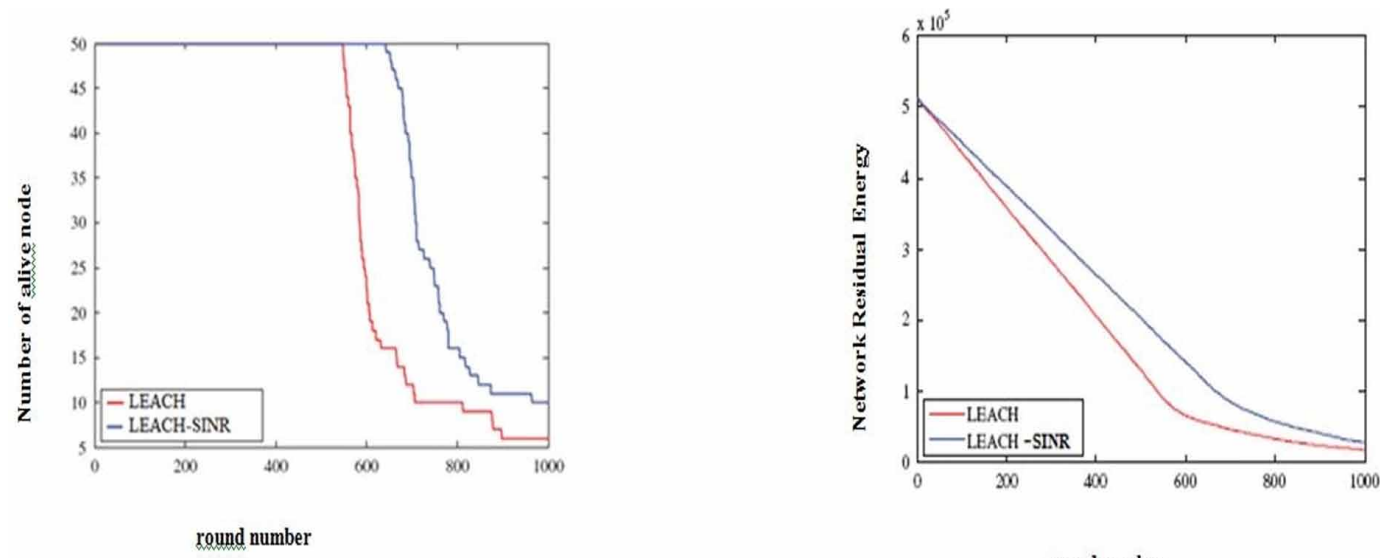

round number

\section{CONCLUSION}

In this paper, the authors started to examine several cross-layer approaches in the first, have presented an enhanced clustering algorithm termed Weighted_Leach in the second, and presented cross layer interaction algorithm in the end. The concept of cross-layer provides a wide field of information exchange between layers.

Still using the notion of least hop count to select a path may not be suitable for WSNs having high mobility and bad environmental conditions making some obstacles, which leads to varying distance between nodes and thus leading to frequent link failure in paths. This lead to the motivation for proposing this work, in which local information's node like distance between nodes, RSSI, SINR and residual energy are taken in consideration during election $\mathrm{CH}$ process at MAC layer protocol and their adaptation to network layer by the cross-layer approach.

The new strategy based competitiveness in clustering algorithm at Mac Layer and jointly coupled with routing algorithm at Network layer give saving energy efficient for WSN communication and high lifetime for WSN cluster. 


\section{REFERENCES}

Acampo, A.S. \& Naghshineh, M. (1994). Control and quality-of-service provisioning in high speed microcellular networks. IEEE Personal Comm. Mag., 36-43.

Bayarkli, S., \& Erdogan, S. Z. (2012). Genetic algorithm based energy efficient clusters (GABEEC) In Wireless Sensors Networks. Procedia Computer Science, 10, 247-2540. doi:10.1016/j.procs.2012.06.034

Blake, S.,Black, D., Carlson, M., Davies, E., Wang, Z., Weiss, W,(1998). An architecture for differentiated services. IETF RFC 2475.

Braden, Clark, \& Shenker. (1994). Integrated services in the Internet architecture: an overview. IETF RFC 1633.

Ekici, Gu \& Bozdag. (2006). mobility based communication in WSN. IEEE commun. Mag., 44, 56-62.

Hortos. (2009). Analytical Models of Cross-Layer Protocol Optimization in Real-Time Wireless Sensor Ad Hoc Networks. Associates in Communications Engineering Research and Technology (ACERT),Ad Hoc Networks First International Conference, ADHOCNETS.

Kawadia, V., \& Kumar, P. R. (2005). A cautionary perspective on cross-layer design. IEEE Wireless Communication Magazine, 12(1), 3-11. doi:10.1109/MWC.2005.1404568

Nikaein, N., \& Bonnet, C. (2003). Layered quality of service model for routing in mobile ad hoc networks. Proc. WMAN.

Nikaein, N., Bonnet, C., Moret, Y., \& Rai, I. A. (2002). 2LQoS - two-layered quality of service model for reactive routing protocols for mobile ad hoc networks. Proc. SCI - 6th World Multi conf. on Systemics, Cyber. and Informatics.

Ramachandran, B., \& Shanmugavel, S. (2008). Received Signal Strength-based Cross-layer Designs for Mobile Ad HocNetworks. IETE Technical Review. Published by Medknow., 25(4), 192-200.

Rappaport, T. S., Annamalai, A., Buehrer, R. M., \& Tranter, W. H. (2002). wireless communication: Past event and future perspective. IEEE Communications Magazine, 40(5), 148-161. doi:10.1109/MCOM.2002.1006984

Shan, Dong, Liao, Shao, Gao, \& Gao. (2013). Research on Improved LEACH Protocol of Wireless Sensor Networks. Przeglad Elektrotechniczny China, 89, 75-77.

Sheta, Mahmoud, Abdelwahab, \& Elaraby. (2015). Analysis and Implementation of Optimized Energy-Based CH Selection. Academic Press.

Sun, L., Li, J., \& Chen, Y. (2005). Wireless Sensor Network. Tsinghua University Press of Technology.

Xu, J., \& Qin, D. (2013). A New LEACH-Based Routing Clustering Protocol in WSN. Journal of Information and Computational Science, 10(18), 18. doi:10.12733/jics20102553

Ouchker Elmekki is a researcher in Wireless sensor networks at high school of technology RITM-ESTC/CEDENSEM, University Hassan II. Area of Interest: Computer Sciences, Network Administration, Cloud Computing, Telecommunication, Network Security.

Maizate Abderrahim, PhD, UH2C, EST, RITM-ESTC/CED-ENSEM, University Hassan II, Casablanca, Morocco. Area of Interest: Computer Sciences, Network Administration, Cloud Computing, Telecommunication, Network Security.

Ouzzif Mohamed (PhD), Professor, RITM-ESTC/CED-ENSEM, University Hassan II, Casablanca, Morocco. Area of Interest: Computer Sciences, Network Administration, Cloud Computing, Telecommunication, Network Security. 